\title{
Investigation on Optimum Parameters of Six-Port Reflectometers
}

\author{
Kamil Staszek
}

\begin{abstract}
In this paper a general investigation on the uncertainty of reflection coefficient measurements with the use of six-port reflectometer is presented. A wide range of both power measurement uncertainty and limited dynamics of the power meters utilized in the six-port systems are incorporated. Based on the conducted analysis the optimum parameters of six-port reflectometer are derived, which provide the lowest possible measurement uncertainty distribution for all reflection coefficients of passive devices.
\end{abstract}

Index Terms-Multiport measurement technique, six-port reflectometer, reflection coefficient, measurement uncertainty, power meters, dynamics of power range.

\section{INTRODUCTION}

Multiport measurement technique is a well-known solution for measurements of complex scattering parameters. Multiport systems feature simple structure, since they are composed of passive power distribution network (multiport), power meters and signal source [1]-[3]. Hence, they are easy to integrate and are adequate for high frequencies [4], [5]. Due to these advantages multiport measurement systems found a wide range of application from radars [6] or microwave receivers [7], [8] to systems for material properties monitoring [9], [10].

A key component of each multiport system is passive power distribution network, which provides signal distribution from signal source to device under test (DUT) and to the applied power meters. This distribution scheme defines general systems' ability to measure complex scattering parameters and can be used for the measurement uncertainty estimation, as shown in [11], where measurement uncertainty of reflection coefficient for a number of different multiports is examined. It is presented that in general systems with higher number of power meters exhibit lower uncertainty, however, such systems are more complicated. Therefore, the most common systems are sixports, which feature the minimum number of power detectors needed for unambiguous measurement. Moreover, by careful design of a six-port the resulting measurement uncertainty can be low [12].

In this paper a new investigation on six-port reflectometers' measurement uncertainty is presented. It extends the investigation reported in [11] by taking into account limited dynamic range of power meters utilized in a six-port system, which in [11] was assumed infinite. As a

Manuscript received January 9, 2019; revised March 15, 2019. This work was supported by the National Science Centre, Poland under grant no. 2016/23/D/ST7/00481

The author is with AGH University of Science and Technology, Krakow, Poland (e-mail: kamil.staszek@agh.edu.pl). result the optimum parameters of a six-port from the measurement uncertainty perspective are found depending on the power meters' dynamics range.

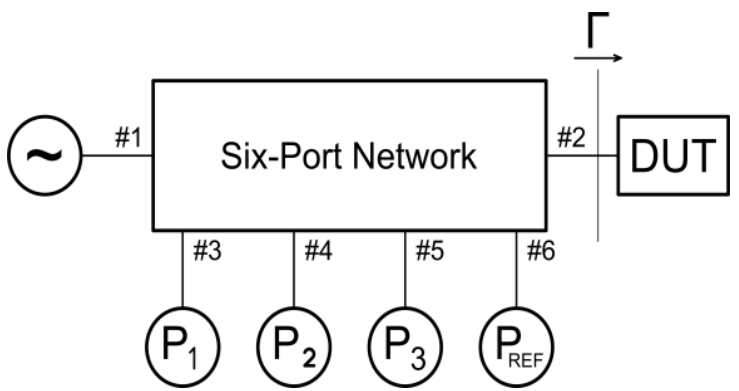

Fig. 1. General block diagram of a six-port reflectometer.

\section{GENERAL PRINCIPLE OF A SIX-PORT REFLECTOMETER}

A generic block diagram of a six-port reflectometer is shown in Fig. 1. It is composed of a passive power distribution network (six-port) to which are connected: signal source (port \#1), DUT (port \#2), three power meters $\mathrm{P}_{1}-\mathrm{P}_{3}$ (ports \#3-\#5), and reference power meter $\mathrm{P}_{\mathrm{REF}}$ (port \#6). The relation between the reflection coefficient $\Gamma$ measured at the measurement port \#2 and the power readings $P_{1}-P_{3}$ and $P_{R E F}$ is as follows [1]:

$$
p_{i}=\frac{P_{i}}{P_{R E F}}=q_{i}\left|\frac{1+A_{i} \Gamma}{1+A_{0} \Gamma}\right|^{2}
$$

where $q_{i}, A_{i}$, and $A_{0}$ are system's constants needed to be found in a calibration procedure prior to actual measurements, and $i=1,2,3$. It is worth mentioning that term $A_{0}$ in (1) refers to the impedance match seen at the measurement port of the six-port. Since six-ports usually exhibit a good impedance match at their measurement ports (port \#2) it can be assumed that $A_{0} \approx 0$. Then, the reflection coefficient $\Gamma$ being the solution of (1) can be interpreted geometrically as an intersection point of three circles on a complex plane, as illustrated in Fig. 2. The location of these circle centers is equal to:

$$
c_{i}=-\frac{1}{A_{i}}
$$

where as their radii can be expressed as:

$$
r_{i}=\sqrt{\frac{p_{i}}{q_{i}\left|A_{i}\right|^{2}}}
$$




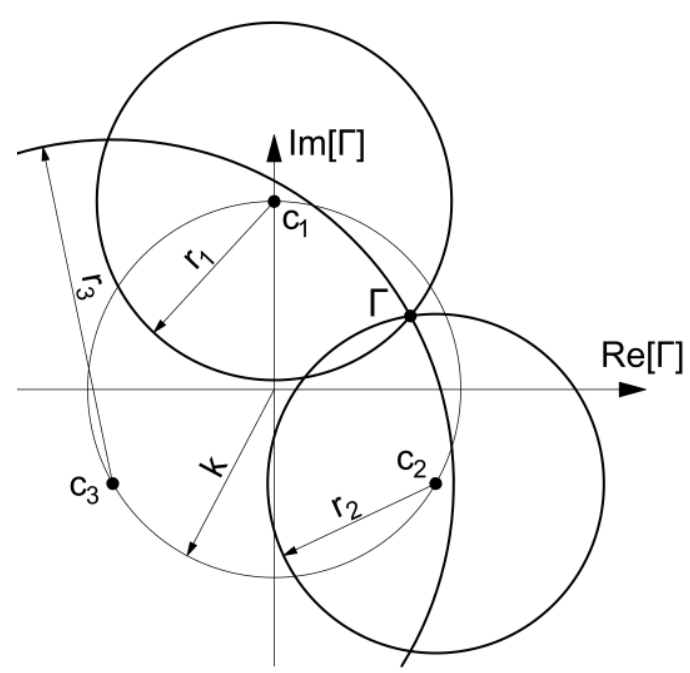

Fig. 2. Geometrical interpretation of a reflection coefficient measurement with the use of six-port reflectometer as intersection point of three circles on a complex plane.

It can be observed that the circle centers distribution $c_{i}$ results from topology of the six-port and the radii $r_{i}$ are proportional to the normalized measured power $P_{i} / P_{R E F}$. Circle centers distribution shown in Fig. 2 is composed of three points arranged uniformly around the complex plane origin at the distance $k$ called the magnitude of circle centers distribution:

$$
k=\left|c_{1}\right|=\left|c_{2}\right|=\left|c_{3}\right|
$$

with $120^{\circ}$ of angular separation:

$$
\arg \left[\frac{c_{1}}{c_{2}}\right]=\arg \left[\frac{c_{2}}{c_{3}}\right]=\arg \left[\frac{c_{3}}{c_{1}}\right]=120^{\circ}
$$

what according to [11] and [12] provides the optimum measurement uncertainty for all reflection coefficients having magnitudes not exceeding unity $(|\Gamma| \leq 1)$. However, in both [11] and [12] the assumed dynamics of power measurement is infinite. Therefore, to investigate the optimum circle centers distribution with limited dynamics of power measurement, the analysis presented in the following section can be conducted.

\section{MEASUREMENT UNCERTAINTY ANALYSIS FOR SIX-PORT REFLECTOMETERS}

According to the mentioned geometrical representation, the reflection coefficient $\Gamma$ is an intersection point of three circles on a complex plane. However, in practice the power readings are deteriorated by power measurement uncertainty $\delta_{P}$, which deviates circles radii $r_{i}$. The maximum and minimum circles' radii $r_{i}^{\max }$ and $r_{i}^{\text {min }}$, respectively, can be then derived as:

$$
\begin{aligned}
& r_{i}^{\max }=r_{i} \cdot 10^{\frac{\delta_{P}}{20}} \\
& r_{i}^{\min }=r_{i} \cdot 10^{-\frac{\delta_{P}}{20}}
\end{aligned}
$$

which are shown in Fig. 3. Since each given radius $r_{i}$ can take a value from $r_{i}^{\min }$ to $r_{i}^{\max }$, all three circles do not intersect in a single point (genuine $\Gamma$ value), but only in close proximity. For instance, assuming that all $r_{i}$ deteriorate to the respective $r_{i}^{\max }$, one can obtain circles as illustrated in Fig. 4. Although they do not intersect in a single point, one can derive three common chords (red lines in Fig. 4), which for three circles provide a single point socalled "radical center" $\Gamma$ ', being a solution of (1) for deteriorated power readings. Since a six-port provides three circles, one can obtain $2^{3}=8$ combinations of $r_{i}^{\max }$ and $r_{i}^{\min }$, resulting in an 8 -element set $\left\{\Gamma_{1}, \ldots, \Gamma_{8}{ }^{\prime}\right\}$. Having these values, the measurement uncertainty $\Delta_{\Gamma}$ for the considered magnitude of circle centers distribution $k$, genuine value of reflection coefficient $\Gamma$, and the assumed power measurement uncertainty $\delta_{P}$ can be calculated as:

$$
\Delta_{\Gamma}\left(k, \Gamma, \delta_{P}\right)=\max _{n}\left|\Gamma-\Gamma_{n}^{\prime}\right|
$$

where $n=1, \ldots, 8$.

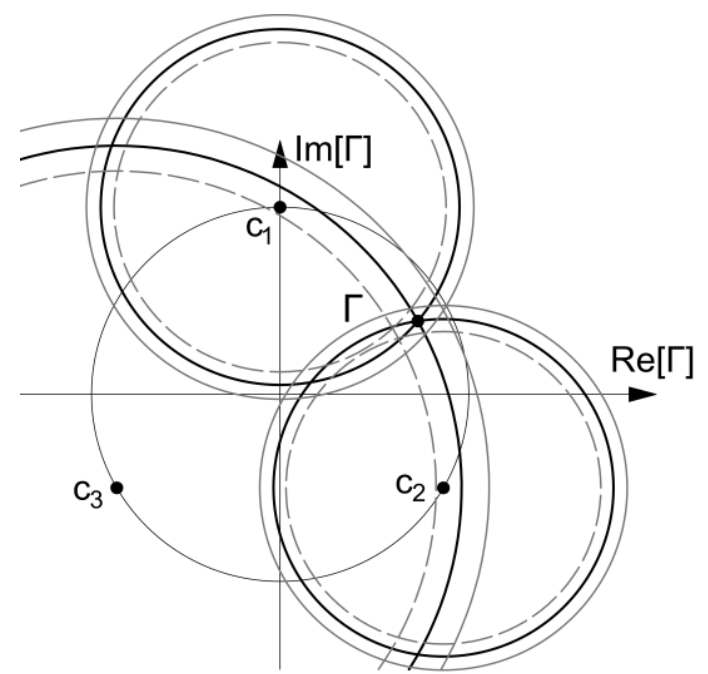

Fig. 3. Influence of power measurement uncertainty $\delta_{P}$ on the circles' radii $r_{i}: r_{i}^{\max }-$ solid gray lines, $r_{i}^{\min }-$ dashed gray lines.

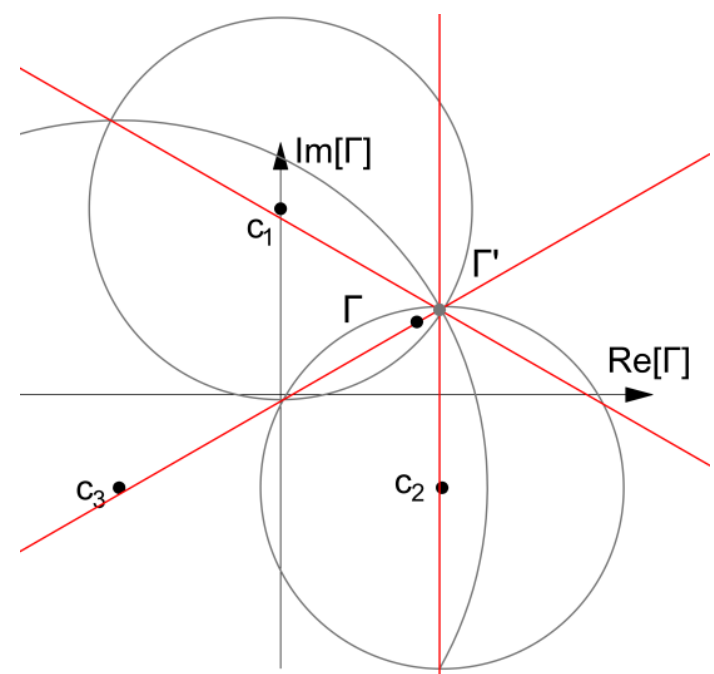

Fig. 4. Reflection coefficient $\Gamma^{\prime}$ calculated using power readings affected by power measurement uncertainty.

The analysis presented above is based on power measurement uncertainty $\delta_{P}$. To make the investigation more complete the impact of limited dynamics range of power measurement $D_{P}$ can be introduced. For simplification the maximum measured power can be assumed to be $P_{i}^{\max }=1 \mathrm{~mW}(0 \mathrm{dBm})$, which requires the coefficients $q_{i}$ in (1) to be equal to: 

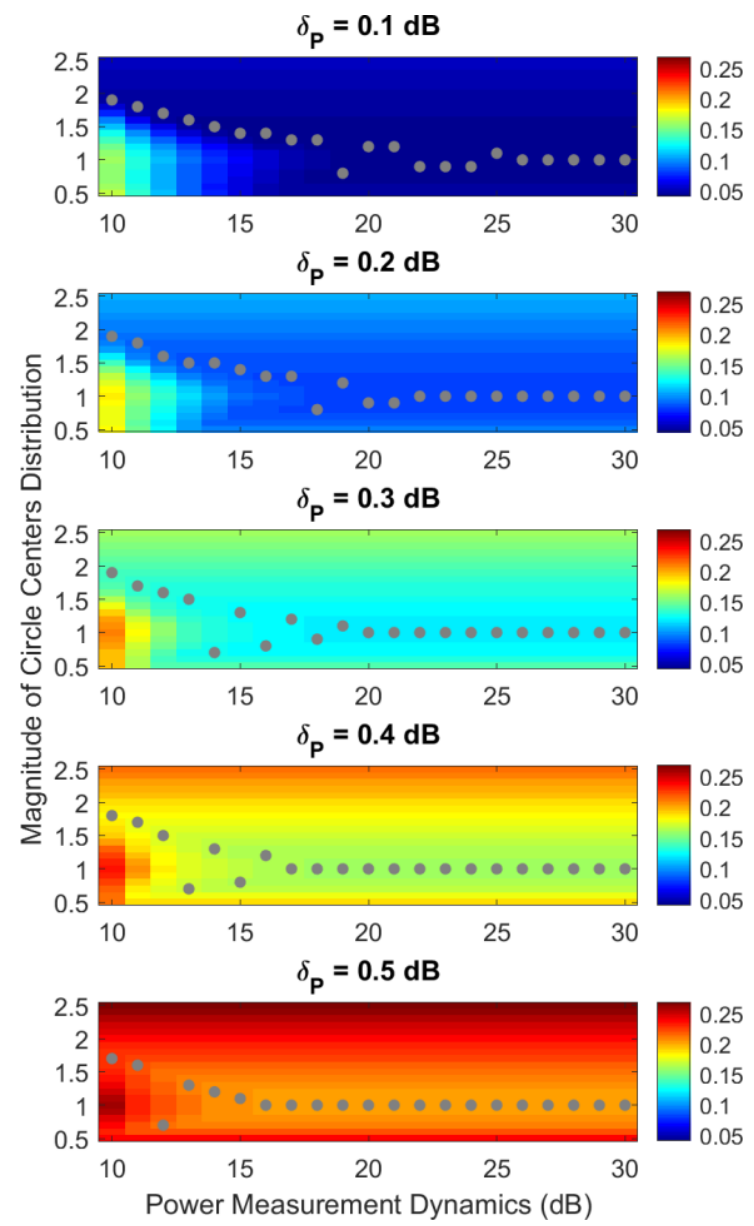

Fig. 5. The maximum value of the measurement uncertainty $\Delta_{\Gamma}$ for all reflection coefficients for which $|\Gamma| \leq 1$ calculated for five different values of power measurement uncertainty $\delta_{P}$, dynamics range of power measurement $D_{P}$, and magnitude of circle centers distribution $k$. Gray dots indicate the minimum values of $\Delta_{\Gamma}$ for given $\delta_{P}$ and $D_{P}$.

$$
q_{i}=\frac{1}{(k+1)^{2}}
$$

Then the minimum measured power $P_{i}^{\min }$ is equal to:

$$
P_{i}^{\min }=10^{-\frac{D_{P}}{10}}
$$

where $D_{P}$ is expressed in $\mathrm{dBm}$. Hence, to estimate the measurement uncertainty $\Delta_{\Gamma}$ including the limited dynamics of the measured power $D_{P}$, the minimum circles' radii $r_{i}^{\min }$ should be recalculated as follows:

$$
r_{i}^{\min }=\max \left[\sqrt{\frac{P_{i}}{P_{R E F}} \frac{10^{-\frac{\delta_{P}}{20}}}{q_{i}\left|A_{i}\right|^{2}}}, \sqrt{\frac{P_{i}^{\min }}{P_{R E F}} \frac{1}{q_{i}\left|A_{i}\right|^{2}}}\right]
$$

\section{Derivation of the Optimum Magnitude of Circle CENTERS DISTRIBUTION $k$}

In order to find the optimum magnitude of circle centers distribution $k$ described by (4) and (5) for six-port reflectometers, the measurement uncertainty investigation presented in Section III has been performed for $k$ varying from 0.5 to 2.5 , five different power measurement uncertainties $\delta_{P}$ in the range from $\pm 0.1 \mathrm{~dB}$ to $\pm 0.5 \mathrm{~dB}$, and power measurement dynamics $D_{P}$ taking values from $10 \mathrm{~dB}$ to $30 \mathrm{~dB}$. For each set of the mentioned parameters the measurement uncertainty $\Delta_{\Gamma}$ has been calculated for all reflection coefficients having magnitudes not exceeding 1. Then, in the obtained measurement uncertainty distribution the maximum and average values have been found and presented in Fig. 5 and Fig. 6, respectively. Additionally, in both Fig. 5 and Fig. 6 the minimum values of the maximum and average $\Delta_{\Gamma}$, respectively, are marked with gray dots, which show the optimum magnitude of circle centers distribution $k_{\text {opt }}\left(\delta_{P}, D_{P}\right)$ for the chosen $\delta_{P}$ and $D_{P}$. For better illustration, the measurement uncertainty $\Delta_{\Gamma}$ calculated for the maximum and the minimum dynamics range $D_{P}$ and for the magnitude of circle centers distribution $k$ equal to 1 and 2 is presented in Fig. 7.
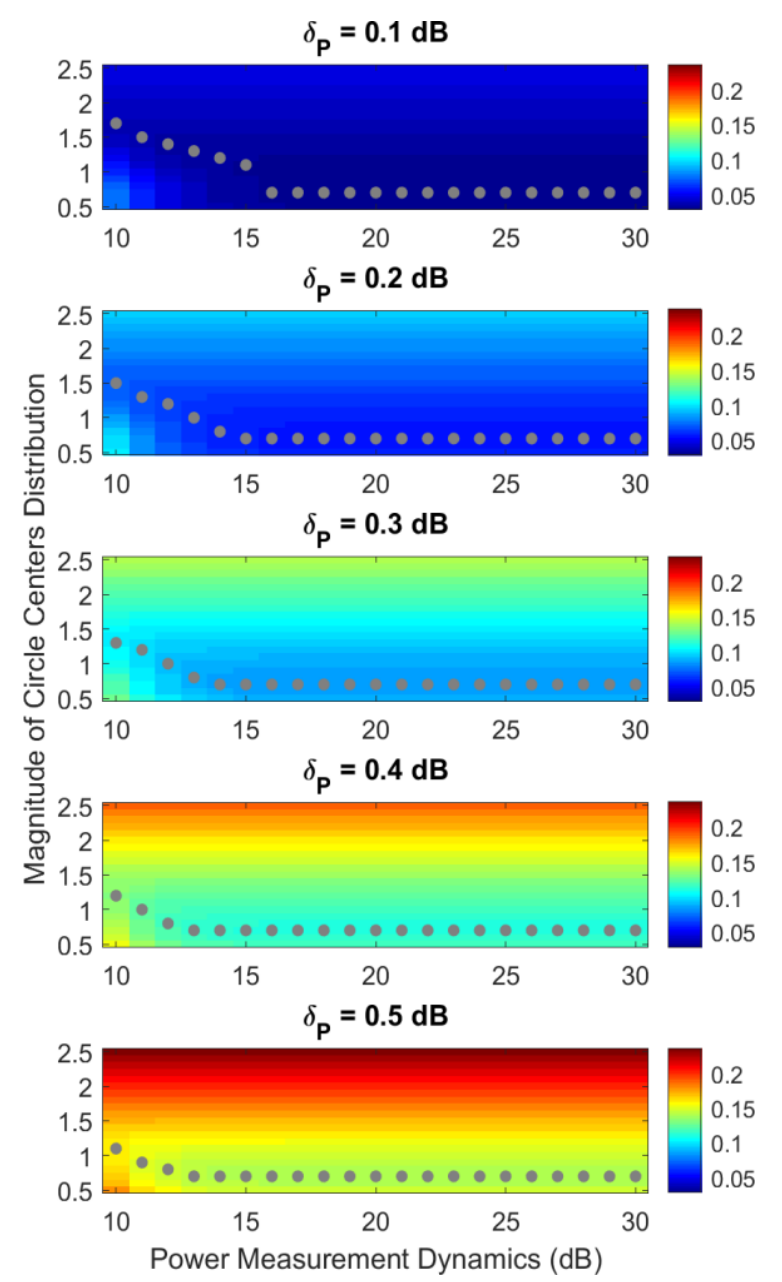

Fig. 6. The average value of the measurement uncertainty $\Delta_{\Gamma}$ for all reflection coefficients for which $|\Gamma| \leq 1$ calculated for five different values of power measurement uncertainty $\delta_{P}$, dynamics range of power measurement $D_{P}$, and magnitude of circle centers distribution $k$. Gray dots indicate the minimum values of $\Delta_{\Gamma}$ for given $\delta_{P}$ and $D_{P}$.

It can be observed that $k_{\text {opt }}$ depends on dynamics range of measured power $D_{P}$, whereas impact of $\delta_{P}$ on $k_{\text {opt }}$ is insignificant. It results from the fact that for low dynamics i.e., low ratio of the maximum and minimum radii $r_{i}$ circle centers cannot be located close to the circumference of the unitary circle (for which $|\Gamma|=1$ ). On the other hand, for $k=$ 1 the dynamics range of measured power goes to infinity, as the minimum circles' radii $r_{i}$ are equal to zero. However, in the obtained results it is clearly seen that the dynamics range $D_{P}$ equal to $20 \mathrm{~dB}$ is sufficient, and a further increase of $D_{P}$ does not provide any improvement in measurement quality. Therefore, assuming that dynamics range $D_{P}=$ 
$20 \mathrm{~dB}$ is not an excessive requirement, it can be stated that the optimum magnitude of circle centers distribution $k$ is equal to 1 .

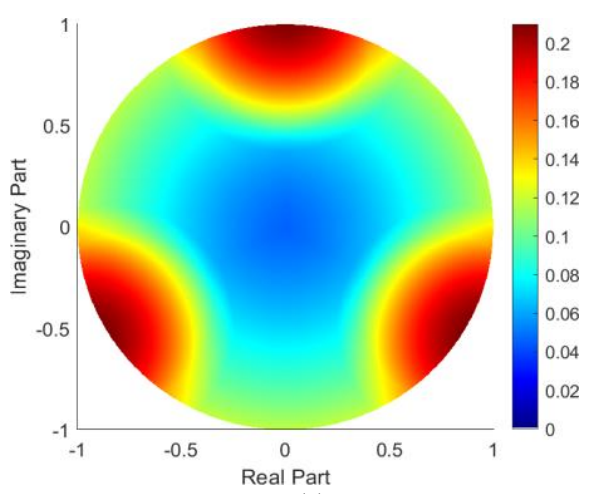

(a)

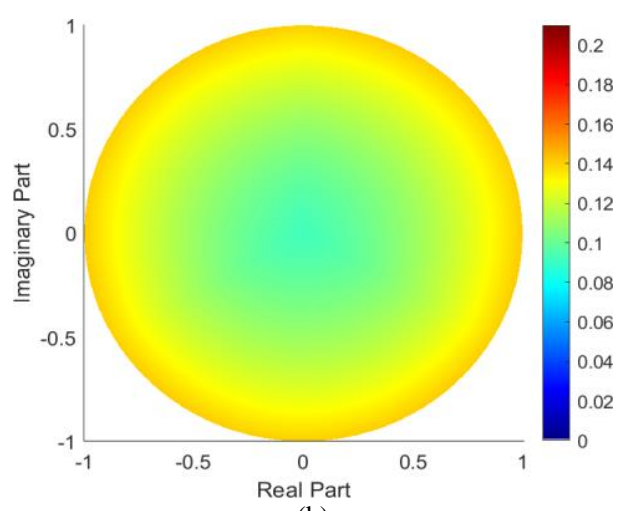

(b)

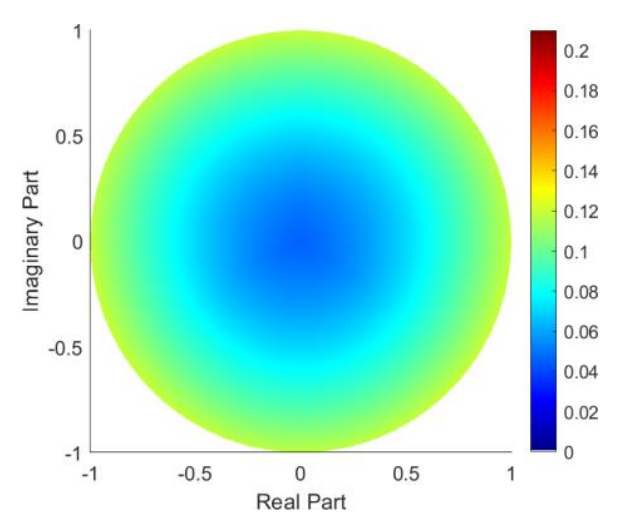

(c)

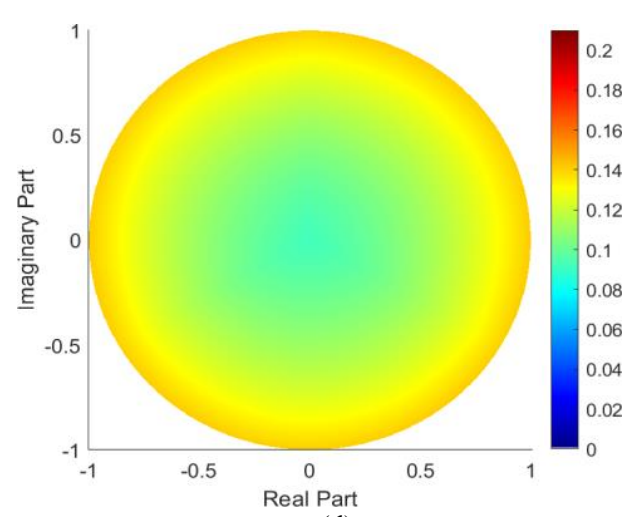

(d)

Fig. 7. Distribution of the reflection coefficient's measurement uncertainty $\Delta_{\Gamma}$ calculated for the circle centers distributions described by (4) and (5) for $\delta_{P}= \pm 0.3 \mathrm{~dB}$ and different magnitude of circle centers distribution $k$ and dynamics range of the measured power $D_{P}: k=1$ and $D_{P}=10 \mathrm{~dB}(\mathrm{a}), k=2$ and $D_{P}=10 \mathrm{~dB}(\mathrm{~b}), k=1$ and $D_{P}=30 \mathrm{~dB}(\mathrm{c})$, and $k=2$ and $D_{P}=30 \mathrm{~dB}(\mathrm{~d})$.

\section{CONCLUSIONS}

In this paper a comprehensive analysis of the measurement uncertainty of six-port reflectometers has been presented. In the given investigation both power measurement uncertainty and limited dynamics range of the power meters utilized in a six-port measurement system have been taken into account. The obtained results have allowed for deriving the optimum six-port network's parameters providing the minimum uncertainty for measurements of reflection coefficients having magnitudes not exceeding unity. In future work the presented investigation will be extended for multiport reflectometers featuring higher number of power meters and the corresponding circle centers $c_{i}$.

\section{REFERENCES}

[1] G. F. Engen, "The six-port reflectometer: An alternative network analyzer," IEEE Trans. Microw. Theory Techn., vol. 25, no. 12, pp. 1075-1080, Dec. 1977.

[2] C. A. Hoer, "Performance of a dual six-port automatic network analyzer," IEEE Trans. Microwave Theory and Tech., vol. 27, no. 12 pp. 993-998, Dec. 1979.

[3] S. Jia, "New application of a single six-port reflectometer," Electronic Letters, vol. 20, no. 22, pp. 920-922, Oct. 1984.

[4] B. Laemmle, K. Schmalz, J. Borngraeber, J. C. Scheytt, R. Weigel, A. Koelpin, and D. Kissinger, "A fully integrated $120 \mathrm{GHz}$ six-port receiver front-end in a $130 \mathrm{~nm}$ SiGe BiCMOS technology," in Proc. IEEE 13th Topical Meeting on Silicon Monolithic Integrated Circuits in RF Systems SiRF 2013, Austin, USA, January 2013, pp. 129-131.

[5] B. Laemmle, G. Vinci, L. Maurer, R. Weigel, and A. Koelpin, “A 77$\mathrm{GHz} \mathrm{SiGe}$ integrated six-port receiver front-end for angle-of arrival detection," IEEE Journal of Solid-State Circuits., vol. 47, no. 9, pp. 1966-1973, Sept. 2012.

[6] F. Barbon, G. Vinci, S. Lindner, R. Weigel, and A. Koelpin, “A sixport interferometer based micrometer-accuracy displacement and vibration measurement radar," in Proc. Int. Microw. Symp. IMS 2012, Montreal, Canada, June 2012, pp. 1-3.

[7] A. O. Olopade, A. Hasan, and M. Helaoui, "Concurrent dual-band six-port receiver for multi-standard and software defined radio applications," IEEE Trans. Microw. Theory Techn., vol. 61, no. 12 pp. 4252-4261, Dec. 2013.

[8] S. O. Tatu, E. Moldovan, K. Wu, and R. G. Bosisio, "A new direct millimeter-wave six-port receiver," IEEE Trans. Microwave Theory and Tech., vol. MTT-49, no. 12, pp. 2517-2522, Dec. 2001.

[9] K. Staszek, I. Piekarz, J. Sorocki, S. Koryciak, K. Wincza, and S. Gruszczyński, "Low-cost microwave vector system for liquid properties monitoring," IEEE Trans. Indust. Electron., vol. 65, no. 2, pp. 1665-1674, February 2018.

[10] K. Kim, N. Kim, S-H. Hwang, Y-K. Kim, and Y. Kwon, "A miniaturized broadband multi-state reflectometer integrated on a silicon MEMS probe for complex permittivity measurement of biological material," IEEE Trans. Microw. Theory Techn., vol. 61, no. 5, pp. 2205-2214, May 2013.

[11] K. Staszek, S. Gruszczynski, and K. Wincza, "Theoretical limits and accuracy improvement of reflection coefficient measurements in sixport reflectometers," IEEE Trans. Microw. Theory Techn., vol. 61, no. 8, pp. 2966-2974, August 2013.

[12] K. Staszek, S. Gruszczyński, and K. Wincza, "Six-port reflectometer providing enhanced power distribution," IEEE Trans. Microw. Theory Techn., vol. 64, no. 3, pp. 939-951, March 2016.

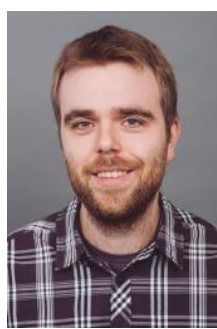

Kamil Staszek received the M.Sc. and Ph.D degrees in electronics engineering from the AGH University of Science and Technology, Cracow, Poland, in 2011 and 2015, respectively. $\mathrm{He}$ is currently with the Department of Electronics, AGH University of Science and Technology. His main research interests include multiport measurement techniques and design of the broadband passive components. He has coauthored 31 journals and 26 conference scientific papers. 